\title{
Editorial: Artificial Intelligence in Insurance and Finance
}

\author{
Glenn Fung ${ }^{1}$, Luisa F. Polania ${ }^{2}$, Sou-Cheng T. Choi ${ }^{3,4 \star}$, Victor $W u^{5}$ and Lawrence $M a^{6}$ \\ ${ }^{1}$ American Family Mutual Insurance Company, Madison, WI, United States, ${ }^{2}$ Palo Alto Networks, Santa Clara, CA, United States, \\ ${ }^{3}$ Kamakura Corporation, Honolulu, HI, United States, ${ }^{4}$ Department of Applied Mathematics, Illinois Institute of Technology, \\ Chicago, IL, United States, ${ }^{5}$ Ensembl Inc., Chicago, IL, United States, ${ }^{6}$ Hong Kong Blockchain Society, Hong Kong, China
}

Keywords: finance, insurance, risk, algorithm, neural network, transformer, bitcoin, chatbot

\section{Editorial on the Research Topic}

Artificial Intelligence in Insurance and Finance

\section{INTRODUCTION}

Artificial intelligence (AI) has become a phenomenon and caught researchers in almost all domains by surprise with its overwhelming success accompanied by unprecedented accuracies, sometimes even surpassing human experts. The finance and insurance sectors are no exceptions to this AI revolution, especially considering the significant amount of historical data, structured and unstructured, available in most financial and insurance companies. Another motivating factor is the evolving expectation of customers for frictionless and on-demand services, which brings not only challenges but also significant opportunities for applying AI. In the field of insurance, AI promises to reshape claims, underwriting, distribution, and pricing. In the field of finance, $\mathrm{AI}$ is having a seismic impact on robo-advisory, fraud prediction, trading strategies, risk assessment, and chatbots, to name a few.

In this context, this research topic brings together 11 papers that have developed new theoretical or applied models employing AI in a variety of financial and insurance problems. We introduce them in the order of the first authors' last names.

*Correspondence:

Sou-Cheng T. Choi schoi32@iit.edu

Specialty section: This article was submitted to Mathematical Finance,

a section of the journal

Frontiers in Applied Mathematics and

Statistics

Received: 14 October 2021

Accepted: 29 October 2021

Published: 01 December 2021

Citation:

Fung G, Polania LF, Choi S-CT, Wu V and Ma L (2021) Editorial: Artificial Intelligence in Insurance and Finance. Front. Appl. Math. Stat. 7:795207. doi: 10.3389/fams.2021.795207

\section{PAPERS IN THIS RESEARCH TOPIC}

Acharya and Fung used state-of-the-art object detection deep learning architectures for extracting vehicle mileage from odometer images taken by mobile devices. Despite availability of commercial solutions for license plates and VIN recognition from images, there are no existing commercial solutions for odometer mileage extraction from images. The authors have also tested empirically the proposed system in unseen odometer images taken in the wild and have achieved satisfactory performance, meeting requirements needed for real-life application in the insurance industry.

Inspired by DNA sequencing and natural language processing, Cheong et al. used historical daily close prices of financial assets and mapped those with positive returns into a sequence, e.g., (AB) represents assets $\mathrm{A}$ and $\mathrm{B}$ with price increases in a day. By such temporal-spatial sequences from every week, the authors tested against random combinations and dynamically discovered assets whose prices often rise together over consecutive days, thereby informing short-term, likely profitable trading decisions.

In the study of Dixon and London, a novel neural network architecture called the $\alpha$-recurrent neural networks ( $\alpha$-RNNs) was developed for non-stationary time series forecasting. In a nutshell, 
$\alpha$-RNNs apply exponentially smoothing to the hidden layers of RNNs and are unconditionally stable with potentially infinite memory. Simple statistical tests suffice to configure $\alpha$-RNNs. Using minute-frequency bitcoin prices and high-frequency futures tick data, the authors demonstrated that $\alpha$-RNNs achieved similar out-sample accuracies as substantially more complex models including gated recurrent units or long shortterm memory models (LSTMs).

Fong et al. developed an innovative insurance application for early, automatic detection of product defects from online customer reviews. The modeling pipeline consists of recurrent neural networks for predicting negative sentiments and the presence of defects; followed by a topic discovery model for clustering negative reviews with defects into similar problems. The application was developed with home products for early intervention and more effective cost management of product defects but is suitable for various artifacts covered by insurance plans.

Fouque and Zhang considered a challenging multiplayer game, in which all players are indistinguishable, and each person seeks to minimize the same objective function of action over time, subject to a stochastic differential equation with delayed effect from an earlier state, given initial conditions. Because there is no closed-form solution, the authors designed two numerical algorithms for estimating an optimal trajectory of controls using neural networks. Last, the authors prove the existence and uniqueness of optimality under certain conditions.

Gupta et al. developed a supervised algorithm that generates task-optimized word embeddings for natural language processing (NLP) applications. Unlike traditional word embeddings that are optimized at the word level, this new algorithm produces embeddings at the sentence-level using a weighted average of an available pre-trained word-level embedding. This allows for more targeted applications of NLP to specific domains and, thus, better performance because the user can inject their weights accordingly. The authors also performed numerical experiments to demonstrate the performance of the algorithm.

Jiang briefly outlined a machine learning approach with LASSO for the challenging problem of forecasting corporate mergers using a large number of annual reports Form10-K filed with the U.S. Securities and Exchange Commission. The unstructured text was preprocessed with NLP techniques and transformed to a high-dimensional term frequency-inverse document frequency matrix. Several potentially fruitful directions are discussed for future research.

Nuti et al. developed a deterministic Bayesian Decision Tree algorithm, applicable to regression and classification problems. It eliminates the need for sampling and pruning. In particular, the algorithm generates a greedy-modal tree (GMT). GMT is salient because models become explainable, an important component and often prerequisite in finance and medicine. The authors tested the new algorithm against various standard benchmarks, demonstrating comparable performance against other existing techniques, showing that accuracy does not have to be sacrificed for explainability.

Bidirectional Encoder Representations from Transformers (BERT) is one of the most advanced AI models for natural languages that have emerged in recent years. Yu et al. leveraged BERT's pre-trained language models to efficiently build a closed-domain chatbot for hierarchical classification of over 380 intents that arise from more than 22,000 questions of financial customers. The article also presents a thorough treatment of out-of-vocabulary words. Finally, model class probabilities are randomly sampled with Monte Carlo methods for computing confidence intervals.

$\mathrm{Yu}$ investigated three information criteria (traditional AIC, $\mathrm{BIC}$, and information complexity-based ICOMP) to assess truncated operational risk models. The performances of using the three information criteria to distinguish various fat-tailed distributional models such as Champernowne, Frechet, lognormal, and Weibull distributions were first examined using simulation studies. The author then studied a use case beginning with model fitting and model validation, followed by value-at-risk estimation, and ended up with model selection using various information criteria on the basis of fraud risk data coming from retail banking of Chinese banks.

The article by Zhang et al. focuses on stock price prediction by leveraging sentiment information from tweets as an additional feature. This is achieved by using a conditional generative adversarial network, where the generator is formed by a LSTM network, whereas the discriminator is formed by a multilayer perceptron. The authors showed through experiments that their approach outperformed state-of-the-art methods based on LSTMs and traditional methods, such as linear multiple regression, $\mathrm{K}$-nearest neighbors, and autoregressive integrated moving average.

\section{CONCLUSION}

In a nutshell, the papers in this research topic illustrate how AI is comprehensively transforming the way financial and insurance businesses operate and interact with their consumers and markets. In particular, deep learning networks are playing an important role in this transformation. For example, many of the articles in this research topic make extensive use of recurrent neural networks to model time series and text data. Similarly, generative adversarial networks and faster region-based convolutional neural networks are also used by some of the articles in this research topic.

It is worth highlighting that the data used in some of the articles go beyond finance and insurance data and extend to social media data, such as tweets. This is motivated by the interplay between social sentiment and financial market movement. It is also worth mentioning that this topic is rich in AI applications to financial and insurance areas, including but not restricted to, financial forecasting, merger activity, financial service chatbots, and risk assessment.

In closing, we would like to express our appreciation to the reviewers for their high-quality feedback and timely responses.

\section{AUTHOR CONTRIBUTIONS}

All authors listed have made a substantial, direct, and intellectual contribution to the work and approved it for publication. 


\section{ACKNOWLEDGMENTS}

The authors would like to thank all editors and reviewers for the Special Topic.

Conflict of Interest: GF was employed by American Family Mutual Insurance Company. LP was employed by Palo Alto Networks. S-CC was employed by Kamakura Corporation. VW was employed by Ensembl Inc.

The remaining author declares that the research was conducted in the absence of any commercial or financial relationships that could be construed as a potential conflict of interest.
Publisher's Note: All claims expressed in this article are solely those of the authors and do not necessarily represent those of their affiliated organizations or those of the publisher, the editors, and the reviewers. Any product that may be evaluated in this article, or claim that may be made by its manufacturer, is not guaranteed or endorsed by the publisher.

Copyright (๑) 2021 Fung, Polania, Choi, Wu and Ma. This is an open-access article distributed under the terms of the Creative Commons Attribution License (CC BY). The use, distribution or reproduction in other forums is permitted, provided the original author(s) and the copyright owner(s) are credited and that the original publication in this journal is cited, in accordance with accepted academic practice. No use, distribution or reproduction is permitted which does not comply with these terms. 